\title{
MYXEDEMA MADNESS: AN INTRIGUING CASE OF DEPRESSION IN HYPOTHYROIDISM
}

\author{
NAVIN PATIL ${ }^{1}$, KARTHIK N RAO ${ }^{2 *}$, BALAJI $0^{1}$, HUMERA NAAZ ${ }^{2}$, GAYATHRI BAIJU ${ }^{2}$, ANANDA NAIK ${ }^{3}$, HARGUN \\ SINGH SIRA ${ }^{4}$
}

${ }^{1}$ Department of Pharmacology, Kasturba Medical College, Manipal University, Manipal, Karnataka, India. ${ }^{2}$ Department of General Medicine, Kasturba Medical College, Manipal University, Manipal, Karnataka, India. ${ }^{3}$ Department of General Medicine, Melaka-Manipal Medical College, Manipal University, Manipal, Karnataka, India. ${ }^{4}$ Kasturba Medical College, Manipal University, Manipal, Karnataka, India. Email: karthikrao85@gmail.com

Received: 11 April 2017, Revised and Accepted: 17 May 2017

ABSTRACT

Hypothyroidism is a clinical syndrome due to deficiency of thyroid hormone. Thyroid hormone is responsible for maintaining the brain hemostasis and helps in central nervous system global development. There have been studies on the association of psychiatric illness with thyroid deficiency and strikingly found to be presenting feature of hypothyroidism in many patients. Literature evidence also says that underlying hypothyroidism may lead to failure of psychiatric treatment. Hence, we report a case of hypertensive patient on medications started with antidepressants and antipsychotics after being diagnosed of depression but was found to be resistant to psychiatric medications and later successfully treated with levothyroxine for underlying hypothyroidism with myxedema.

Keywords: Hypothyroidism, Psychosis, Affective disorder, Levothyroxine.

(C) 2017 The Authors. Published by Innovare Academic Sciences Pvt Ltd. This is an open access article under the CC BY license (http://creativecommons. org/licenses/by/4. 0/) DOI: http://dx.doi.org/10.22159/ajpcr.2017.v10i8.19081

\section{INTRODUCTION}

Hypothyroidism is a clinical condition characterized by a deficiency of thyroid hormone presenting with different manifestations ranging from metabolic derangements to global developmental abnormalities. Stereotypical cluster of symptoms includes hair loss, fatigue, dry skin, intolerance to cold, menstrual irregularities, and constipation. Usually, signs of hypothyroidism include bradycardia, facial puffiness, slow speech, non-pitting type of pedal edema, and delayed deep tendon reflexes. Psychiatric disorders are known to accompany hypothyroidism and diagnosis usually ranges from mild mental status impairment to that of severe psychosis and mood disorders [1]. Hence, we present a case of hypothyroidism presenting itself has depression not responding to psychiatric medications in a 57-year-old hypertensive male in a tertiary care hospital in Southern India.

\section{CASE REPORT}

Informed consent was obtained from the patient. A 57-year-old male, a known hypertensive on tablet amlodipine $5 \mathrm{mg}$ twice daily, was referred from a psychiatrist in view of increased fatigue and drowsiness noticed for past 1 week. The patient was on tablet escitalopram $10 \mathrm{mg}$ for depression for 3 months and tablet quetiapine $50 \mathrm{mg}$ for $1 \mathrm{month}$ for hallucinations due to psychosis. General examination revealed an obese male, with coarse dry skin of extremities, macroglossia, periorbital edema, and bipedal edema. Vital signs revealed pulse rate of $54 / \mathrm{min}$ and temperature of $38^{\circ} \mathrm{C}$. He was drowsy, but arousal to calling was present and followed simple commands but with slowness in the gross and fine motor activities. There were mild slurring of speech and delayed relaxation of the ankle jerks (Woltman's sign). Hypothyroidism was suspected and thyroid-stimulating hormone levels were $>100$ IU, and serum creatine kinase levels were also markedly elevated 830 (normal up to 80). A diagnosis of severe hypothyroidism was made, and levothyroxine loading dose with parenteral hydrocortisone was administered. Escitalopram and quetiapine were stopped. His symptoms improved remarkably with thyroxine replacement. His antidepressants and antipsychotics were discontinued and dose of thyroxine adjusted after repeating thyroid function tests.

\section{DISCUSSION}

Hypothyroidism and psychiatric illness have a close association and are said to be not infrequent. Hypothyroidism is one of the most common etiology overlooked for behavioral and cognitive changes in a patient. Forgetfulness, fatigue, mental slowness, and emotional lability are the most predominant symptoms, and sometimes, hypothyroid patients are misdiagnosed of primary psychiatric illness in the first place due to presenting symptoms leading to delay as well as precipitation of underlying thyroid illness. Depression is the most common affective disorder seen in hypothyroid patients [2]. The brain tends to use thyroid hormone very differently when compared to other organs in the body, and thyroid hormone is required for the development of central nervous system as well as maintenance of hemostasis. $\mathrm{T}_{3}$ receptors are present in high concentrations in the hippocampus and amygdala influencing the neural activity. $\mathrm{T}_{4}$ hormone exerts its neurobehavioral action through an effect on various neurotransmitters. In a study, it was found that hypothyroid rats were found to have increased amount of tyrosine hydroxylase activity and increased dopamine levels in comparison to hyperthyroid rats [3-5]. Incidence of psychotic disorders associated with hypothyroidism ranges from 5 to $15 \%$ with traditionally patients presenting with lassitude and lethargy as in initial manifestation [6]. Depression being the most common affective disorder associated with hypothyroidism, the incidence of depression associated with clinical hypothyroidism is $40 \%$ [7]. Although the incidence of depression with subclinical hypothyroidism is not known, refractory depression is known to be very common in patients with subclinical hypothyroidism with incidences ranging up to 50\% [8]. Treatment-resistant depression usually responds to thyroid supplementation with or without laboratory investigation revealing altered thyroid status. Various theories have been proposed on the association of depression with hypothyroidism. Main and foremost one is the involvement of serotonergic system, and it has been found that reduced levels of serotonin were found in the brain in case of hypothyroidism [9]. Other hypotheses include reduced or inhibited activity of Type 25 'deiodinase enzyme leading to reduced conversion of $\mathrm{T}_{4}$ to $\mathrm{T}_{3}$ in the brain and depression is related to brain catecholamine deficiency in hypothyroid patients [9] 
The treatment is usually replacement of thyroid hormone and psychiatric disturbances usually get corrected as the endocrinopathy is corrected. The use of antidepressants is usually sought only after correcting the thyroid status. Moreover, the use of quetiapine can aggravate a preexisting overt severe hypothyroidism and precipitate into a myxedema coma. The risk of quetiapine per day causing hypothyroidism is about $0.4 \%$ with about half of them requiring thyroid replacement therapy [10]. In this case, after diagnosing the patient with depression, he was started with antidepressants along with antipsychotics. However, the patient never responded. Other signs and symptoms suggestive of underlying hypothyroidism with myxedema coma. Hence, levothyroxine and corticosteroids were started, and antidepressants and antipsychotics were stopped. The patient improved after 2 weeks.

\section{CONCLUSIONS}

This patient presented with severe myxedema and pre-coma like features. The depression was secondary to hypothyroidism and could have been treated effectively if thyroid function tests were obtained before starting antidepressant medications. Moreover, addition of quetiapine and escitalopram precipitated and accelerated the rate of progression of hypothyroidism. Myxedema coma is a medical emergency and can be fatal if not diagnosed early and treated effectively. Prompt recognition of the near fatal myxedema and early initiation of thyroxine resulted in effective "cure" of his depression.

\section{REFERENCES}

1. Rita JA, Nambi S. A case of hypothyroidism induced recurrent depressive disorder. Int J Pharm Pharm Sci 2015;7(11):397-8.

2. Pomeranze J, King EJ. Psychosis as first sign of thyroid dysfunction. Geriatrics 1966;21(6):211-2.

3. Thompson CC, Potter GB. Thyroid hormone action in neural development. Cereb Cortex 2000;10(10):939-45.

4. Dratman MB. Cerebral versus peripheral regulation and utilization of thyroid hormones. In: Joffe RT, Levitt AJ, editors. The Thyroid Axis and Psychiatric Illness. Washington, DC: American Psychiatric Press, Inc.; 1993. p. 3-94.

5. Ruel J, Faure R, Dussault JH. Regional distribution of nuclear T3 receptors in rat brain and evidence for preferential localization in neurons. J Endocrinol Invest 1985;8(4):343-8.

6. Hall RC. Psychiatric effects of thyroid hormone disturbance. Psychosomatics 1983;24(1):7-11, 15-8.

7. Pies RW. Women, mood, and the thyroid. Women Psychiatry Health 1995;4:4-10.

8. Howland RH. Thyroid dysfunction in refractory depression: Implications for pathophysiology and treatment. J Clin Psychiatry 1993;54(2):47-54.

9. Cleare AJ, McGregor A, O'Keane V. Neuroendocrine evidence for an association between hypothyroidism, reduced central 5-HT activity and depression. Clin Endocrinol (Oxf) 1995;43(6):713-9.

10. Chari S. The lesson from a yellow psychotic patient. Hosp Med 2002;63(6):370-1 